\title{
Childhood Deaths with a Co-Morbidity of Diarrhea and Severe Malnutrition: A Brief Insight in an Urban Critical Care Ward in Dhaka, Bangladesh
}

Md Shakil Hossain ${ }^{1}$, Sufia Islam², Mohammod Jobayer Chisti ${ }^{3}$

\begin{abstract}
Background and aim: Although co-morbidity of childhood diarrhea and severe malnutrition is very common with high mortality, data on predicting factors for deaths in diarrheal under-five children also having severe malnutrition are very limited in medical literature. The aim of this study was to evaluate the clinical predicting factors for death in diarrheal under-five children with severe malnutrition.

Methods: The study was designed as a prospective analysis from retrospective data of diarrheal children (case control design) which were collected from electronic database of the hospital of the International Center for Diarrheal Disease Research, Bangladesh (icddr,b) from mid-September 2011 to mid-September 2012. The cases were severely malnourished diarrheal children under the age of five years who died in the intensive care unit and the controls were those who survived. Comparison of clinical characteristics among the cases and the controls were made.

Results: There were 32 cases from the ICU and 1790 controls including 253 from the ICU. The median (inter-quartile range) age (months) of the cases compared to the controls was significantly lower [8.0 (4.1, 14.1) vs. 10.0 (6.1, 17.2); $p=0.050]$. The cases more often had pneumonia (OR 3.40, 95\% CI 1.48-7.66, $p<0.001)$ with respiratory distress (OR 30.06, 95\% CI 11.47-77.67, $p<0.001)$, frequently presented with the features of clinical sepsis (OR 52.22, 95\% CI 24.02-127.68; $p<0.001$ ), less often received Oral Rehydration Salt (ORS) at home (OR 0.07, 95\% CI 0.03-0.14; $p<0.001)$, and more often had lower Z-score for weight for age [(mean -5.42 \pm standard deviation 1.35) vs. (mean -4.14 \pm standard deviation 1.28); $p<0.001]$ compared the controls.

Conclusion: The brief results of the data suggest that severely malnourished diarrheal children presenting with younger age with a history of lack of intake of ORS at home, extreme under-weight, pneumonia with respiratory distress or clinical sepsis are at higher risk of death. This re-emphasizes the importance of identification of these simple clinical parameters which may help in early aggressive management of these children and underscores the importance of the requirement of extensive mass media education in encouraging the adequate intake of ORS during diarrhea in order to reduce their morbidity and deaths.
\end{abstract}

Key Words: Childhood Death, Diarrhea, Malnutrition.

\section{Introduction}

Severe Malnutrition is one of the most common causes of morbidity and mortality among children in developing countries including Bangladesh ${ }^{1,2}$. Among the global deaths in children under five years of age, about $48 \%$ occurred in different forms of malnutrition $(19 \%$ were due to underweight, $14.5 \%$ to stunting, and $14.6 \%$ to wasting) $)^{3}$. Although the prevalence rates of childhood malnutrition are slowly regressing in Asia, the highest prevalence can still be

1. Md Shakil Hossain, M. Pharm, Research Fellow, Clinical Service (CS), International Centre for Diarrhoeal Disease Research, Bangladesh (icddr,b), Dhaka, Bangladesh and Department of Pharmacy, East West University, Dhaka, Bangladesh.

2. Sufia Islam, M. Pharm, Ph.D, Associate Professor and Head, Department of Pharmacy, East West University, Dhaka, Bangladesh.

3. Mohammod Jobayer Chisti, MBBS, MMed (Paed), Scientist, Centre for Nutrition and Food Security \& Clinical Lead, ICU, Centre for Nutrition and Food Security (CNFS), icddr,b, Dhaka, Bangladesh.

Corresponding Author:

Dr. Mohammod Jobayer Chisti, MBBS, MMed (Paed)

Scientist, Centre for Nutrition and Food Security \&

Clinical Lead, ICU, Dhaka Hospital

International Centre for Diarrhoeal Disease Research, Bangladesh (icddr,b) 68 Shaheed Tajuddin Ahmed Sarani, Mohakhali, Dhaka 1212, Bangladesh E-mail: chisti@icddrb.org found in South Asia ${ }^{1}$. Malnutrition has been responsible, directly or indirectly, for $54 \%$ of the global annual deaths among children under five $\mathrm{f}^{4}$. The immediate causes of malnutrition and child death are mutually reinforcing conditions of inadequate dietary intake and infectious disease; the underlying causes are household food insecurity, inadequate health service and absence of healthy environment ${ }^{1}$. According to national guidelines, 2008; the death rate among children hospitalized for severe acute malnutrition in Bangladesh was as high as 15 percent $^{5}$. The Dhaka hospital of International Centre for Diarrheal Disease and Research, Bangladesh (icddr,b) runs the Clinical Research and Service Centre (CRSC) in Dhaka, Bangladesh, which treats more than 120000 patients for diarrheal disease each year ${ }^{6}$. The overall mortality rate in the CRSC is only $0.45 \%$, but the mean mortality rate among severely malnourished children with diarrhea is about $15 \%$, and most of these deaths occur within the 48 hours of admission 7 . Death is even higher at intensive care unit (ICU) of this setting with this co-morbidity of diarrhea and severe malnutrition ${ }^{8}$. These two contributes each other and associated with a number of risks for death. However, data are limited on the predicting factors of deaths in such children at critical care set ups. It is thus important to identify the simple predicting factors for death to initiate 
prompt management in order to reduce deaths in such children. The purpose of our analysis, using a case-control design, was to evaluate the predicting factors for death in severely malnourished children below 5 years of age attending the ICU of the Dhaka Hospital of icddr,b with diarrheal diseases.

\section{Methods}

\section{Study population \& Site}

The study was conducted at the Dhaka hospital of icddr,b. This hospital provides care and treatment to over 120000 diarrheal patients, with or without associated health problems, each year. It also conducts research on enteric and other common infectious diseases and malnutrition, and provides training on case management and research methodology. Under-five children constitute over $60 \%$ of the total patients, and vast majority of the patients come from the poor socio-economic background of urban and peri-urban Dhaka, the capital of Bangladesh.

\section{Study Design}

The study was designed as a prospective analysis from retrospective data of patients collected from the 'SHEBA', an online database from icddr,b. For the collection of data a questionnaire was prepared which was given to the programmed analyst of the hospital for assembling the data. All under-five pediatric patient suffering from diarrhea having severe malnutrition and admitted in the ICU of the Dhaka Hospital of icddr,b from September 2011 to September 2012 were enrolled in the study. Comparison of simple clinical features was made among the children who died \& survived. Severe malnutrition was defined following WHO anthropometry ${ }^{9}$.

\section{Patient management}

Treatment received by the study children in the ICU/HDU/ARI ward have been described elsewhere?.

\section{Measurements}

Case report forms (CRF) were developed, pretested, and finalized for data acquisition. Death was the primary outcome and characteristics analyzed include age, clinical sepsis ${ }^{10}$, pneumonia $^{11}$, respiratory distress, ORS intake at home, and Z score for weight for age.

\section{Analysis}

All data were entered into SPSS for Windows (version 15.0; SPSS Inc, Chicago) and Epi-Info (version 6.0, USD, Stone Mountain, GA). Differences in the proportions were compared by the Chi-square test. For normally distributed data, the differences of means were compared by Student's t-test and Mann-Whitney test was used for comparison of data that were not normally distributed. A probability of less than 0.05 was considered statistically significant. Strength of association was determined by calculating odds ratio (OR) and their 95\% confidence intervals (CIs).

\section{Results}

A total of 1822 children enrolled during the study period and among them $32(2 \%)$ died in the ICU and 1790 survived including 253 in the ICU. The median (inter-quartile range) age (months) of the children who died compared to the survivors was significantly lower $[8.0(4.1,14.1)$ vs. 10.0 $(6.1,17.2) ; p=0.05]$. The children who died frequently presented with pneumonia (31\% vs. $12 \%$; OR $3.40,95 \%$ CI $1.48-7.66, \mathrm{p}<0.001)$ with respiratory distress $(28 \%$. $1 \%$; OR $30.06,95 \%$ CI 11.47-77.67, $\mathrm{p}<0.001$ ), often had the features of clinical sepsis (53\%. 2\%; OR 52.22, 95\% CI 24.02-127.68; $\mathrm{p}<0.001$ ), seldom received ORS at home (50\% vs. 94\%; OR $0.07,95 \%$ CI $0.03-0.14 ; \mathrm{p}<0.001)$, and more often had lower $\mathrm{Z}$ score for weight for age [(mean $-5.42 \pm$ standard deviation 1.35 ) vs. (mean $-4.14 \pm$ standard deviation 1.28); $\mathrm{p}<0.001$ ] compared the controls.

\section{Discussion}

This study observed a number of simple clinical predicting factors for death in diarrheal children having severe malnutrition: younger age, pneumonia with respiratory distress, clinical sepsis, less intake of ORS at home, and extreme under-weight. These observations are not surprising at all, however, in spite of decreasing trend of worldwide malnutrition, for the last few decades severe malnutrition remained as one of the greatest contributors of deaths in south Asia, including Bangladesh due to inadequate social and political commitment ${ }^{1}$. Thus, these simple clinical predicting factors for deaths in diarrheal children having severe malnutrition is critically important for the clinicians as well as policy makers to take adequate preventive as well as curative measures.

The observation of clinical sepsis as one of the predicting factors in diarrheal children with severe malnutrition is understandable. Clinical sepsis in severely malnourished children often associated with vasodilatation and capillary leakage ${ }^{12,13,14}$ and leads to poor peripheral microcirculation ${ }^{10}$ with fatal outcome9. The observation of association of less intake of ORS at home with deaths in these children is also understandable. Lack of replacement of purging by taking ORS is often associated with a number of ramifications such as dehydration, shock, hypoglycemia, electrolyte imbalance especially hyponatremia and hypokalemia following convulsion $^{7,11}$ which are highly associated with deaths in such children $^{7,15,16}$. Observation of association of pneumonia with respiratory distress with deaths in such children has also been reported earlier ${ }^{17}$. The higher the severity of malnutrition, the higher the rates of deaths, especially in diarrheal children ${ }^{18}$ and this explains our observation of higher deaths in diarrheal children with extreme malnutrition. Observation of younger age as one of the predicting factors of death in diarrheal children with severe malnutrition has also been reported earlier? .

The main limitation of the analyses is the fewer number of variables which impeded the exploration of potentially more predicting factors associated with deaths in diarrheal children having severe malnutrition.

In conclusion, severely malnourished diarrheal children who present with a history of lack of adequate intake of ORS at home, young age (8 months), extreme under-weight, pneumonia with respiratory distress, and clinical sepsis are prone to deaths. Identification of these simple clinical 
predicting factors may help in early aggressive management of these children and re-emphasizes the importance of further requirement for extensive mass media education in encouraging the adequate intake of ORS during diarrhea in order to reduce their morbidity and deaths.

\section{Acknowledgements}

This research was funded by icddr,b and its donors which provide unrestricted support to icddr,b for its operations and research. Current donors providing unrestricted support include: Australian Agency for International Development (AusAID), Government of the People's Republic of Bangladesh; Canadian International Development Agency (CIDA), Swedish International Development Cooperation Agency (Sida), and the Department for International Development, UK (DFID). We gratefully acknowledge these donors for their support and commitment to icddr,b's research efforts. We also like to express our sincere thanks to SHEBA personnel for their invaluable support during data collection.

\section{References :}

1. Ahmed T, Hossain M, I. SK (2012) Global Burden of Maternal and Child Undernutrition and Micronutrient Deficiencies. Ann Nutr Metab 61 (supplement): 8-17.

2. Chisti MJ, Hossain MI, Malek MA, Faruque AS, Ahmed T, et al. (2007) Characteristics of severely malnourished under-five children hospitalized with diarrhoea, and their policy implications. Acta Paediatr 96: 693-696.

3. Black RE, Allen LH, Bhutta ZA, Caulfield LE, de Onis M, et al. (2008) Maternal and child undernutrition: global and regional exposures and health consequences. Lancet 371: 243-260.

4. Schaible UE, Kaufmann SH (2007) Malnutrition and infection: complex mechanisms and global impacts. PLoS Med 4: e115.

5. National Guidelines for the Management of Severely Malnourished Children in Bangladesh (2008).

6. Chisti MJ, Salam MA, Ashraf H, Faruque AS, Bardhan PK, et al. (2013) Predictors and outcome of hypoxemia in severely malnourished children under five with pneumonia: a case control design. PLoS One 8: e51376.

7. Ahmed T, Ali M, Ullah MM, Choudhury IA, Haque ME, et al (1999) Mortality in severely malnourished children with diarrhoea and use of a standardised management protocol. Lancet 353: 1919-1922.
8. Chisti MJ, Pietroni MA, Smith JH, Bardhan PK, Salam MA (2011) Predictors of death in under-five children with diarrhoea admitted to a critical care ward in an urban hospital in Bangladesh. Acta Paediatr 100: e275-279.

9. Chisti MJ, Salam MA, Ashraf H, Faruque AS, Bardhan PK, et al. (2013) Clinical risk factors of death from pneumonia in children with severe acute malnutrition in an urban critical care ward of bangladesh. PLoS One 8: e73728.

10. Chisti MJ, Duke T, Robertson CF, Ahmed T, Faruque AS, et al. (2012) Clinical predictors and outcome of hypoxaemia among under-five diarrhoeal children with or without pneumonia in an urban hospital, Dhaka, Bangladesh. Trop Med Int Health 17: 106-111.

11. WHO (2006) Pocket book for hospital care of children: guidelines for the management of common illness with limited resources Geneva: World Health Organization. pp. 173-195.

12. Annane D, Bellissant E, Cavaillon JM (2005) Septic shock. Lancet 365: 63-78.

13. Ebrahim GJ (2011) Sepsis, septic shock and the systemic inflammatory response syndrome. J Trop Pediatr 57: 77-79.

14. Chisti MJ, Ahmed T, Faruque AS, Abdus Salam M (2010) Clinical and laboratory features of radiologic pneumonia in severely malnourished infants attending an urban diarrhea treatment center in Bangladesh. Pediatr Infect Dis J 29: 174-177.

15. Samadi AR, Wahed MA, Islam MR, Ahmed SM (1983) Consequences of hyponatraemia and hypernatraemia in children with acute diarrhoea in Bangladesh. Br Med J (Clin Res Ed) 286: 671-673.

16. Chisti MJ, Ahmed T, Bardhan PK, Salam MA (2010) Evaluation of simple laboratory investigations to predict fatal outcome in infants with severe malnutrition presenting in an urban diarrhoea treatment centre in Bangladesh. Trop Med Int Health 15: 1322-1325.

17. Chisti MJ, Salam MA, Hossain MI, Ashraf H, Faruque ASG, et al. (2013) Fatal outcome related risks in severely malnourished children with pneumonia in an urban critical care ward of Bangladesh. . Bangladesh Critical Care Journal 1: 80-85.

18. Roy SK, Buis M, Weersma R, Khatun W, Chowdhury S, et al. (2011) Risk factors of mortality in severely-malnourished children hospitalized with diarrhoea. J Health Popul Nutr 29: 229-235. 